\title{
Reverberation chamber simulation system research on radar battlefield electromagnetic environment
}

\author{
Yuying LIANG ${ }^{a}$, Peng TU ${ }^{b}$, Zhuangzhi HAN ${ }^{c}$, Hao WANG ${ }^{d}$ \\ Department of Electronics and Optics Engineering, Machanical Engineering College, Shijiazhuang \\ 050003, China \\ aemail:Yy_Liang_oec@163.com, bemail:825985189@qq.com, cemail:Zz_Han_oec@163.com, \\ demail: wanghao4577@163.com
} Keywords: Radar; Battlefield Electromagnetic Environment; Reverberation Chamber; Effectiveness
Evaluation; Fault Information

\begin{abstract}
In order to realize the radar electromagnetic environment simulation, improve radar system combat effectiveness. A simulation system based on reverberation chamber concentrating on radar electromagnetic environment was proposed. The system was made by three processing center. According to the measured battlefield electromagnetic environment data, the system radiate electromagnetic waves into reverberation chamber through several signal producing module. By setting transmitting and receiving antenna position and making different stirrer ways in reverberation chamber (RC), to simulate complex radar battlefield electromagnetic environment. In addition, finish radar fault information collection and effectiveness evaluation via the feedback information from test radar during the whole process of simulation.
\end{abstract}

\section{Introduction}

Battlefield electromagnetic came from traditional battlefield environmental elements which stands out in the important position of modern war [1]. The electromagnetic power competition became much fierce. In general, the signal density, forms diversity, dynamic overlap, style complexity is the main performance of electromagnetic environment complexity[2]. Thus, realize radar electromagnetic environment simulation is the key for testing radar performance. At present, radar electromagnetic environment simulation method mainly includes: hardware generation based on actual equipment, software technology based on computer simulation[3,4] and electromagnetic radiation based on simulator[5,6]. The cost was high by using actual equipment. Software simulation method could overcome deficiencies of the first method, but the simulation accuracy was influenced by the model complexity and precision. Simulator had both advantages of the former method thus it became a hot research in recent years. All kinds of simulators emerged in endlessly. In addition, there were specific ones which aimed at radar clutter background[7-9] or other jammers under other conditions[10,11]. However, manufacturing complex electromagnetic environment in a closed chamber method was less involved. So, we put forward a radar battlefield electromagnetic environment simulation system based on reverberation chamber.

\section{The basic principle of reverberation chamber}

Reverberation chamber can be regarded as high quality factor resonance cavity, statistically homogeneous, isotropic, random polarization are its ideal electromagnetic characteristics [12].

Suppose, a location $\vec{r}$ in reverberation chamber has electric field $\vec{E}(\vec{r})$, the coordinate express is:

$\vec{E}=\vec{e}_{x} E_{x}+\vec{e}_{y} E_{y}+\vec{e}_{z} E_{z}$

In the formula $E_{x}=E_{x r}+i E_{x i}, E_{y}=E_{y r}+i E_{y i}, \quad E_{z}=E_{z r}+i E_{z i}$.

In a fully stirred reverberation chamber:

$\left\langle E_{x r}\right\rangle=\left\langle E_{x i}\right\rangle=\left\langle E_{y r}\right\rangle=\left\langle E_{y i}\right\rangle=\left\langle E_{z r}\right\rangle=\left\langle E_{z i}\right\rangle=0$ 


$$
\begin{aligned}
& \left\langle\left|E_{x}\right|^{2}\right\rangle=\left\langle\left|E_{y}\right|^{2}\right\rangle=\left\langle\left|E_{z}\right|^{2}\right\rangle=\frac{16}{3} \pi C=\frac{E_{0}^{2}}{3} \\
& \left\langle\left|E_{x r}\right|^{2}\right\rangle=\left\langle\left|E_{x i}\right|^{2}\right\rangle=\left\langle\left|E_{y r}\right|^{2}\right\rangle=\left\langle\left|E_{y i}\right|^{2}\right\rangle=\left\langle\left|E_{z r}\right|^{2}\right\rangle=\left\langle\left|E_{z i}\right|^{2}\right\rangle=\sigma^{2} \\
& \left\langle\left|E_{x r}\right|\right\rangle=\left\langle\left|E_{x i}\right|\right\rangle=\left\langle\left|E_{y r}\right|\right\rangle=\left\langle\left|E_{y i}\right|\right\rangle=\left\langle\left|E_{z r}\right|\right\rangle=\left\langle\left|E_{z i}\right|\right\rangle=0
\end{aligned}
$$

Formula(2) (5) reflects the isotropic characteristics.

From the angular spectrum function and nature of $\delta$ function:

$$
\left\langle|E(r)|^{2}\right\rangle=4 C \iint_{4 \pi} d \Omega=16 \pi C=E_{0}^{2}
$$

Formula(6) reflects the statistically homogeneous characteristics.

In addition, the time constant $\tau$ of reverberation chamber is also an important parameter:

$$
\tau=\frac{Q}{\omega}
$$

$\mathrm{Q}$ is the quality factor of reverberation chamber; $\omega$ is angular frequency.

Mechanical stirring reverberation chamber can build a stable electromagnetic environment in a very short period of time. Its electromagnetic characteristics are all good properties of complex electromagnetic environment establishment. When the internal space is big enough, it can be used as radar battlefield electromagnetic environment simulator.

\section{Radar battlefield electromagnetic environment simulation system}

Simulation system. Make reverberation chamber as the simulation center of radar battlefield electromagnetic environment, periphery with guide control center, signal generation center and fusion processing center. Block diagram was shown in figure 1:

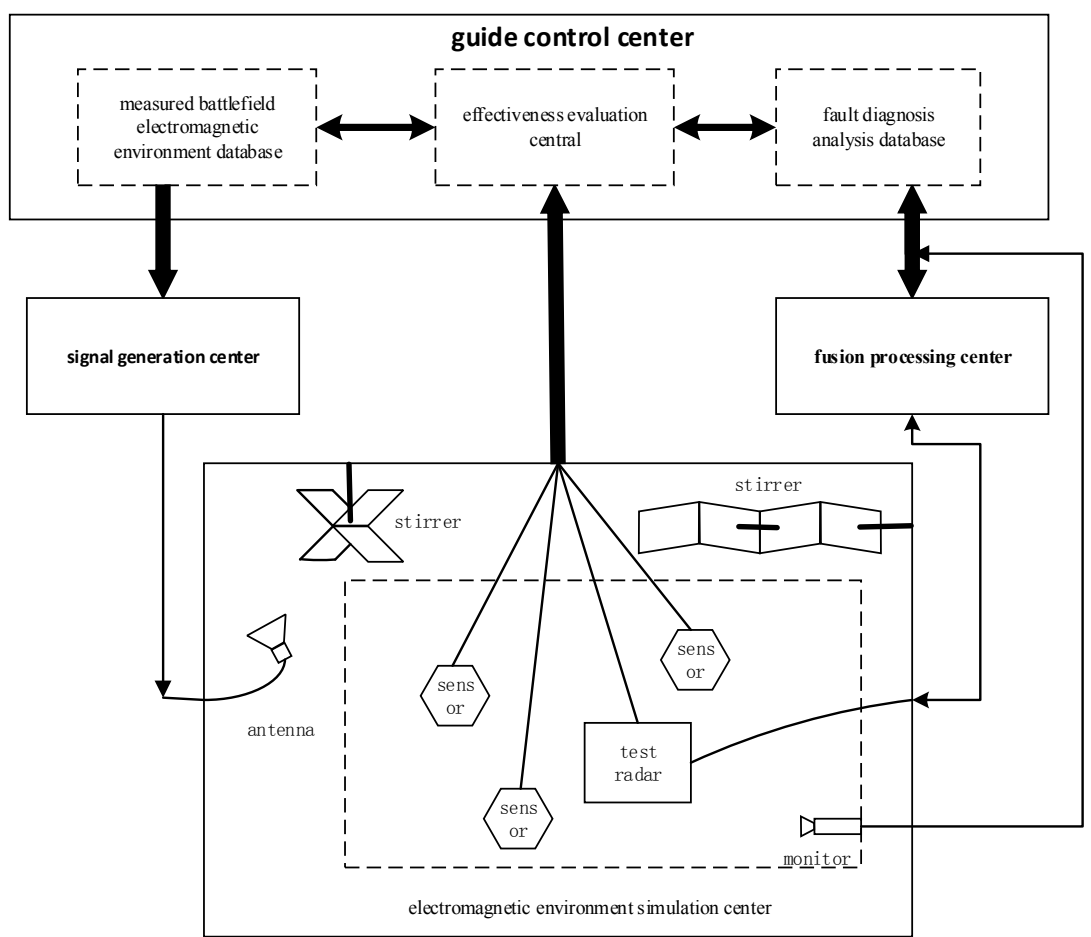

Fig. 1 System block diagram of radar electromagnetic environment simulation

Guide control center. This part was the core of the whole system, mainly included: the measured battlefield electromagnetic environment database, the effectiveness evaluation central and fault diagnosis analysis database. Environment database collected the battlefield electromagnetic environment information, which came from: military maneuver, shooting range training, daily training et al. Environment database extracted electromagnetic environment data indicators from the time, frequency, energy, space "four domain": time domain last degree $t c$, types of signal time vary 
degree $\sigma_{t}$ (time domain index); spectrum possess degree $f_{c}$, spectrum density $\rho_{f}$ (frequency domain index); environmental noise density $\rho_{n}$, power density $\rho_{p}$, number of radiation sources $n$ (energy domain index); airspace coverage $\rho_{s}$, space field intensity $E_{s}$ (space domain index). Effectiveness evaluation central tested the simulation of battlefield electromagnetic environment. Take $t c$, $f_{\mathcal{C}}, \rho_{p}$ and $\rho_{s}$ as evaluation index and give each index different weighting factor $\left(\varepsilon_{i}, 0 \leq \varepsilon_{i} \leq 1\right.$, $\left.\sum_{i=1}^{4} \varepsilon_{i}=1\right)$ according to different battlefield background. Thus we could define electromagnetic environment complexity:

$$
C_{w}=\varepsilon_{1} C\left(t_{c}\right)+\varepsilon_{2} C\left(f_{c}\right)+\varepsilon_{3} C\left(\rho_{p}\right)+\varepsilon_{4} C\left(\rho_{s}\right)
$$

$C\left(t_{c}\right), C\left(f_{c}\right), C\left(\rho_{p}\right), C\left(\rho_{s}\right)$ were the normalized function of $t_{c}, f_{c}, \rho_{p}, \rho_{s}$.

The fault diagnosis analysis database mainly received, classified and stored real-time fault data from fusion processing center, and determined the threat level that electromagnetic environment made on tested radar through the real-time data and monitor information.

Signal generation center. Signal generation center was made by several composable signal producing module. Each module was equivalent to an independent signal source:

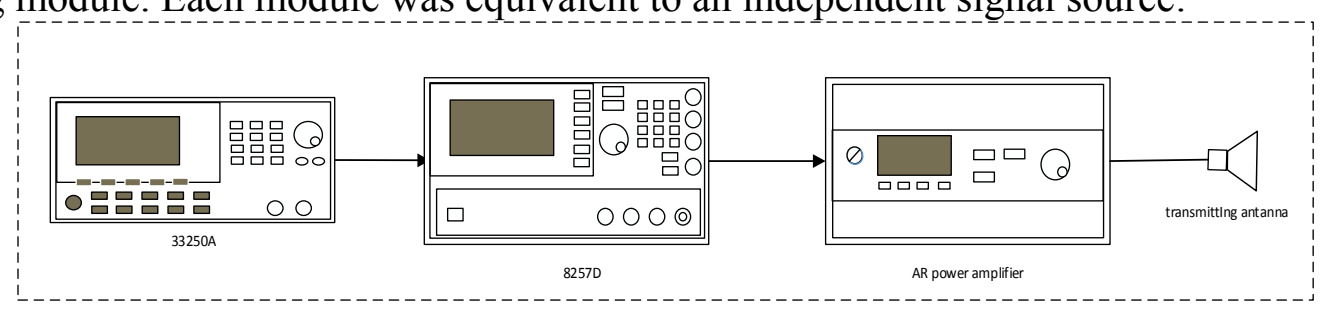

Fig. 2 Signal producing module

We could see in figure 2, the signal emission module mainly consisted of four parts: arbitrary signal generator (33250A), analog signal generator(8257D), AR power amplifier and transmitting antenna. Arbitrary signal generator could make different types of signals. Then the signal flew into analog signal generator that could modulate it into a higher frequency which was greater than reverberation chamber minimal generation frequency. Power amplifier power mainly the modulation signal. Finally the amplified signal was injected into the chamber via transmitting antenna. Modules were independent of each other and guided by electromagnetic environment database, which could simulate different number of radiation sources affecting tested equipment synchronously or asynchronously.

Fusion processing center. This part, like a bridge connecting control center and simulation chamber, mainly dealt with fault information, state information from tested radar. Work flow chart was shown in figure 3:

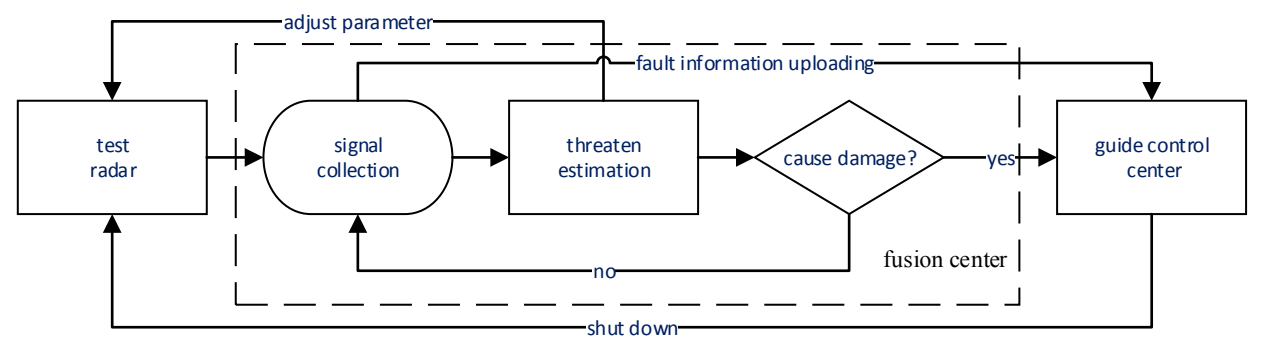

Fig.3 Flow chart of fusion center operation

Fusion center collected information from radar, uploaded real-time fault information to guide center and made threat estimation forecast for radar through the known information and signal processing algorithm. Then feedback the information to radar and adjust its working parameters to confront the effects of electromagnetic environment. Then continue to collect relevant information from radar. When the threat level came to a certain extent, fusion center must judge whether radar would face the danger of damage. If yes, immediately report to the control center, conduct shutdown protection and stop simulation. If no, repeat the process above. 
Electromagnetic environment simulation center. The mechanical stirred reverberation chamber used in this paper had a size of $10.5 m \times 7.9 m \times 4.3 m$, which could place a medium-sized radar or several small-sized ones. Because the reverberation chamber time constant $\tau$ was tiny, its internal electromagnetic environment could reach stability in a very short time. When combat scene changed rapidly, reverberation chamber could response to meet the need of simulation situation quickly. Reverberation chamber shielding performance was good, which could make it insensitive to outer electromagnetic environment. Cavity could easily form a multipath effect, which was consistent with common radar clutter electromagnetic environment. So we could successfully simulate different amplitude distribution characteristics of radar clutter environment. Logarithmic normal distribution radar clutter electromagnetic environment had been simulated as shown in figure 4:

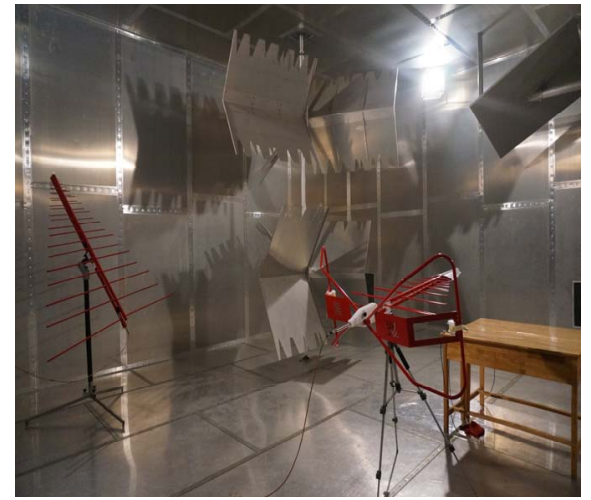

(a) $\mathrm{RC}$ experiment

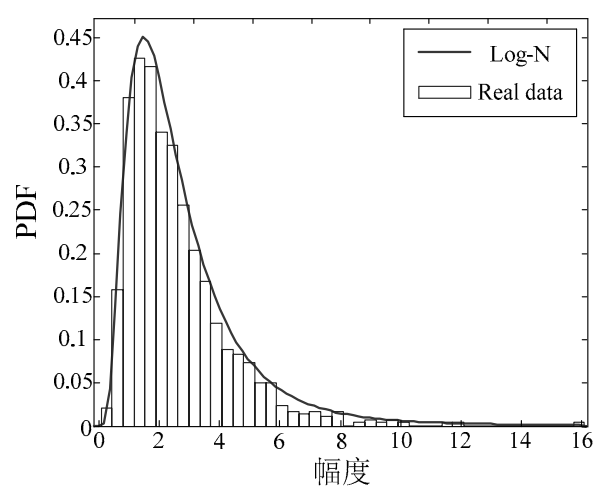

(b) amplitude distributing

Fig.4 Radar clutter electromagnetic environment simulation in RC

Stirrers stayed unmoved, changing the input signal number, form, frequency, power et al in signal generation center that could form a mild complex electromagnetic environment. In addition, when came into continuous stirring condition, the electromagnetic wave in cavity was also stirred, the features of statistically homogeneous, isotropic and random polarization were inspired. This, in turn, could simulate a more complex and changeable radar battlefield electromagnetic environment.

\section{Conclusion}

The successful establishment of radar battlefield electromagnetic environment and realistic simulation of it had an important role in anti-jamming capability training, fault information debugging and comprehensive performance improving. In this paper, a battlefield electromagnetic environment simulation system based on mechanical stirred reverberation chamber had been proposed. System was divided into three levels: first level guide control center was control system, second level signal generation center and fusion center were execution system, third level simulation center was operation system. In coordinated control of the guide center, reverberation chamber could simulate different background of radar battlefield electromagnetic environment. Through sensors and radar, the system evaluated the simulated electromagnetic environment for different aspects.

\section{References}

[1] Tao Shao, Yihua $\mathrm{Hu}$, Liang Shi, et al. Methods for quantitative evaluation of battlefield electromagnetic environment complexity [J]. Electronics Optics \& Control, 2010,17(1):81-84.

[2] Jie Cheng. The design and implementation of signal acquisition and processing system on electromagnetic environment simulation of radar [D]. Nanjing University of Science and Technology, 2013.

[3] Jinhua Xu, Guangbin Liu. Evaluation of battlefield electromagnetic environment effect based on grey analytical hierarchy process [J]. Electronics Optics \& Control, 2010,17(4):14-17. 
[4] Fang Zhang, Jinyan Cai, Yanhui Zhu. Joint modeling and simulation of radar electromagnetic environment [J]. Equipment Environment Engineering, 2009,6(2):86-88.

[5] Yuzhen Gong. Real-time electromagnetic environment simulation and its implementation method [J]. Modern Radar, 2005,27(6):66-68.

[6] Jie Chen, et al. Design and implementation of a novel simulation of complex electromagnetic environment [J]. Information Research, 2009,35(9):22-25.

[7] Huiming Wu. A radar clutter simulator based on PCI bus [J]. Computer Simulation, 2005,22(10):31-34.

[8] Xi Li, Zhiming Pan. A study on the implementation of airborne radar clutter environment monitor [J]. Modern Radar, 2009,31(12):5-9.

[9] Shufeng Gong. Modeling and hardware-in-the-loop simulation for wideband and coherent radar environment clutter [D]. Nanjing University of Aeronautics and Astronautics, 2011.

[10] Xiaoqiang $\mathrm{Wu}$, Dong Zhao, Xipeng Wang. Distributed miniaturization radar jamming technology [J]. Shipboard Electronic Countermeasure, 2010,33(1):32-35.

[11] Yu Zhu, Jingchun Luo, Wei Tian. Research on multi-false-target jamming against netted radar [J]. Electronics Optics \& Control, 2013,20(9):93-98.

[12] Jianjin Ding. Theory, measurement and design of reverberation chamber [D]. Beijing Jiaotong University, 2005. 